\title{
Evaluation of Photosynthetic Characters and Regulation Pattern of Photosynthesis Associated Gene in Two Mulberry Varieties
}

\author{
Yong $\mathrm{Li}^{1,2}$, Cui $\mathrm{Yu}^{2}$, Rongli $\mathrm{Mo}^{2}$, Chao Xiong ${ }^{2}$, Zhixian $\mathrm{Zhu}^{2}$, Xingming $\mathrm{Hu}^{2}$, Chuxiong Zhuang ${ }^{{ }^{*}}$ and Wen Deng ${ }^{2 *}$ \\ ${ }^{1}$ College of Life Sciences, South China Agricultural University, Guangzhou 510642, China \\ ${ }^{2}$ Cash Crops Research Institute, Hubei Academy of Agricultural Sciences, Wuhan 430064, China \\ *Corresponding authors: 68568911@qq.com \\ Received 19 October 2020; Accepted 05 January 2021; Published 25 March 2021
}

\begin{abstract}
Photosynthetic characteristics and expression patterns of the photosynthesis-related genes in the high-yield mulberry variety E'Sang 1 (E1) and normal mulberry variety Husang 32 (H32) were investigated in this study. The observation of daily variation of photosynthesis in E1 and H32 indicated that the peak of net photosynthetic rate $(P \mathrm{n})$ in E1 variety was significantly higher than that in H32 $(P<0.05)$. Meanwhile, the $P$ n- PAR and $P$ n- $C$ i responses of E1 and H32 were evaluated, and the results showed that the carboxylation efficiency and compensation saturation point were much higher in E1 rather than H32. Importantly, the photosystem II actual photochemical efficiency and photochemical quenching coefficient in the leaves of E1 were significantly higher than those in $\mathrm{H} 32(P<0.05)$. Also, the activity of RuBP in E1 was higher than that in $\mathrm{H} 32(P>$ 0.05). Based on the RNA-seq data, a total of 3,356 differentially expressed genes (DEGs) were detected among different time points between E1 and H32. Of these, 1,136 DEGs were involved in the metabolic pathways, including three main photosynthesis-related metabolic pathways (i.e., carbon fixation in photosynthetic organisms, carbon metabolism, and porphyrin and chlorophyll metabolism). Meanwhile, 10 novel DEGs related to photosynthesis were detected, and four potential key genes of them could account for the differences in net photosynthetic rate and yield between H32 and E1. This study could provide important insights into the molecular breeding of mulberry varieties with high photosynthetic efficiency and contribute to understanding the genetic mechanism of photosynthesis. (C) 2021 Friends Science Publishers
\end{abstract}

Keywords: Mulberry; Photosynthetic characteristics; Chlorophyll fluorescence characteristics; Gene expression

\section{Introduction}

Photosynthesis in higher plants is an extremely complex process enabling material production (Wu et al. 2017; Feng et al. 2019). The photosynthesis characteristics of plants have been a hot topic for many years and the genetic mechanisms of better light use efficiency of a plant are important for underlying the photosynthesis characteristics (Shen et al. 2008; Ryu et al. 2019). Morus alba L. is a deciduous tree or shrub. As a traditional feed for silkworm (Bombyx mori L.), mulberry leaves are important materials in the sericulture industry and have great nutritional and medicinal value. Currently, mulberry leaves and mulberries are listed by the Ministry of Health of China as "one of the agricultural products that are both food and medicine". Besides, mulberries have spread throughout the world and are highly praised for their unique flavor and impressive composition of nutrients.

Previously, most photosynthesis studies in mulberry trees mainly focused on the effects of stress and artificial cultivation techniques on their photosynthetic characteristics
(Tezara et al. 1999; Peng et al. 2015; Nemali and Iersel 2019). To our knowledge, little information on photosynthetic characteristics among different mulberry varieties is available. Some studies focused on the effects of abiotic stresses on photosynthesis of mulberry. For instance, Ramanjulu et al. (1998) compared the effects of water stress on photosynthesis of the drought tolerant and sensitive mulberry cultivars and found that some photosynthetic characters were different between two different cultivars. Also, the effects of salinity, waterlogging and thermal stresses on photosynthesis of mulberry varieties were also investigated in previous studies (Agastian et al. 2000; Chaitanya et al. 2003; Yu et al. 2013). To investigate the biological regulation mechanisms of the difference of photosynthesis and yield between two different mulberry varieties under natural growth conditions, the major physiological differences in photosynthesis were compared between the high-yield mulberry variety E'Sang 1 (E1) and normal mulberry variety Husang 32 (H32) (Li et al. 2014). These two varieties have been widely planted in Yangtze river basin of China for many years. Of these, H32 was bred

To cite this paper: Li Y, C Yu, R Mo, C Xiong, Z Zhu, X Hu, C Zhuang, W Deng (2021). Evaluation of photosynthetic characters and regulation pattern of photosynthesis associated gene in two mulberry varieties. Intl J Agric Biol 25:863-872 
in 1978 and E1 is a newly bred variety which has better performance of stress tolerance and yield (Ye et al. 2010). Meanwhile, RNA-seq of leaves in different stages of these two mulberry varieties was conducted to identify the potential key genes related to photosynthesis. Although many RNA-seq studies related to mulberry have been reported (Dai et al. 2015; Wang et al. 2018; Dai et al. 2020), the genetic dynamics of photosynthesis related genes in mulberry were still rarely studied. This study aimed to address two main questions, (1) the physiological difference of photosynthesis in the mulberry varieties and (2) unveiling the gene regulation difference of photosynthesis in the mulberry varieties. This study contributes to underlying the biological mechanism of photosynthetic characteristics between the two different mulberry varieties under natural conditions.

\section{Materials and Methods}

\section{Sampling}

Two mulberry varieties E'Sang1 (E1) and Husang 32 (H32) were cultivated to form middle trunks and planted in 1996 at a density of $133 \times 67 \mathrm{~cm}$ in the Mulberry Germplasm Resource Garden in Hubei Province of China. Test plots with ground leveling and uniform land fertility were selected. Experiments were conducted in triplicate. Three mulberry trees with similar trunk girth, crown diameter, and tree vigor were selected from a test plot for sampling. Pruning was conducted in summer (July and August). Prevention and treatment of mulberry pests and shoot thinning were conducted to ensure a good group structure for leaf production. The soil in the test field was typical yellow-brown soil with moderate fertility and slight acidity. The $\mathrm{pH}$ values ranged from 5.6 to 6.5 , and the organic matter contents were above average. The sampling was performed from 2014 to 2019.

\section{Measurement of photosynthetic characters}

The net photosynthetic rate $\left(P_{\mathrm{n}}\right)$, stomatal conductance $(\mathrm{Gs})$, $\mathrm{Ci}$, and transpiration rate $(\mathrm{Tr})$ of mulberry leaves were determined using an LI-6400XT portable photosynthesis analyzer manufactured by LI-COR (Lincoln, NE, USA) (Chen et al. 2010). Daily variation in photosynthesis was measured. The leaves were selected from three wellilluminated top shoots of mulberry trees. One leaf with normal function was selected from each shoot (from leaf positions 5-7). The measurement period was from 6:00 to 18:00. Measurements were performed once every $2 \mathrm{~h}$, with three replicates; The $P_{\mathrm{n}}$-photosynthetically active radiation (PAR) and $P_{\mathrm{n}}-C_{\mathrm{i}}$ response curves were also evaluated. For $P_{\mathrm{n}}$-PAR response curves, 14 gradients from 0 to 1,800 $\mu \mathrm{mol} /\left(\mathrm{m}^{2} \cdot \mathrm{s}\right)$ were set, and the atmospheric $\mathrm{CO}_{2}$ concentration was $400 \mu \mathrm{mol} / \mathrm{mol}$. The initial slope of the $P_{\mathrm{n}^{-}}$ $P A R$ response curve $\mathrm{d} P_{\mathrm{n}} / \mathrm{d} P A R$ was obtained by linear regression $\left(0-200 \mu \mathrm{mol} /\left(\mathrm{m}^{2} \cdot \mathrm{s}\right)\right)$, which showed the apparent quantum efficiency (AQY). The light compensation point $(L C P)$ and light saturation point $(L S P)$ were calculated using a fitting curve equation $\left(y=a x^{2}+b x+c\right)$ (Peñuelas et al. 1998). The $\mathrm{Pn}-\mathrm{Ci}$ response curves were measured in the same period. Twelve concentrations $(0-1500 \mu \mathrm{mol} / \mathrm{mol})$ were used for $\mathrm{CO}_{2}$ and PAR was $1200 \mu \mathrm{mol} /\left(\mathrm{m}^{2} \cdot \mathrm{s}\right)$. The initial slope $\mathrm{d} P_{\mathrm{n}} / \mathrm{d} C_{\mathrm{i}}$ of the $P n-C i$ response curve was obtained by linear regression $(0-200 \mu \mathrm{mol} / \mathrm{mol})$, which was the carboxylation efficiency (CE). The $\mathrm{CO}_{2}$ compensation point (CCP) and saturation point (CSP) were calculated using the $\mathrm{Pn}$-Ci response curve equation (Zhou et al. 2019).

\section{Chlorophyll fluorescence measurement}

Chlorophyll fluorescence was determined using a fluorescent leaf chamber of the LI-6400 photosynthesis analyzer. Leaf positions and leaves were selected as described above. Firstly, the leaves were completely wrapped with aluminum foil for 12-24 h of shading before the experiment. When mulberry leaves were completely dark-adapted, they were accurately measured from 5:30 to 7:00 in the morning. Each sample was measured 6 times.

During the measurement, the detection light was turned on to determine the minimum initial fluorescence (Fo), and all PSII reaction centers were open. After the Fo was measured, the de-excitation of leaves was achieved using intense saturated pulsed light, and dark-adapted maximum fluorescence (Fm) was measured. Subsequently, the leaves were exposed to continuous photochemically active light $\left[\mathrm{PPFD}=1200 \mu \mathrm{mol} /\left(\mathrm{m}^{2} \cdot \mathrm{s}\right)\right]$ for $30 \mathrm{~min}$ to determine the steady-state fluorescence (Fs). The action light was turned on to provide continuous and appropriate supersaturated light $\left(\mathrm{PPFD}=2000 \mu \mathrm{mol} /\left(\mathrm{m}^{2} \cdot \mathrm{s}\right)\right)$ to illuminate the leaves, and the maximum fluorescence $\left(\mathrm{Fm}^{\prime}\right)$ after light adaptation was obtained. After the measurement, the action and detection lights were turned off and far-red light was applied to measure the light-adapted initial fluorescence (Fo'). Other chlorophyll fluorescence parameters such as photosystem II (PSII) maximum photochemical efficiency (Fv/Fm), PSII actual photochemical efficiency (ФPSII), photochemical quenching coefficient (qP), nonphotochemical quenching (NPQ), and PSII electron transfer rate (ETR) were automatically calculated, and the main chlorophyll fluorescence parameters were calculated as follows:

(1) PSII maximum quantum efficiency: $F v / F m=(F m-F o) / F m$ (Genty et al. 1989)

(2) PSII actual quantum efficiency: $\Phi P S I I=\Delta F / F m^{\prime}=\left(F m^{\prime}-\right.$ Fs)/Fm' (Genty et al. 1989)

(3) Apparent electron transfer rate: $E T R=0.5 \times 0.84 \times \Phi P S I I \times P P F D$ (Demmig et al. 1987)

(4) Photochemical quenching coefficient: $q P=\left(F m^{\prime}-F s\right) /\left(F m^{\prime}-\right.$ $F_{0}$ )

(5) Non-photochemical quenching: $\mathrm{NPQ}=\left(\mathrm{Fm}-\mathrm{Fm}^{\prime}\right) / \mathrm{Fm}^{\prime}$. 


\section{Measurement of physiological and biochemical characters}

Chlorophyll was extracted using phosphate-buffer containing $80 \%$ acetone and analyzed at $646.6 \mathrm{~nm}, 663.6$ $\mathrm{nm}$ and $750 \mathrm{~nm}$, respectively (Porra et al. 1989; Brouwer et al. 2012) using a Spectrophotometer. Rubisco activity was measured by conducting coupled spectrophotometric assays (Kubien et al. 2011).

\section{Statistical analysis}

Mean values of five values were calculated using Microsoft Office Excel 2007. The ANOVA (analysis of variance) was performed with SPSS software (SPSS, IL, USA).

\section{RNA-seq of two mulberry varieties}

RNA extraction and RNA-seq: Leaf samples of two mulberry varieties were collected at the time of peak and trough of the daily variation curve of photosynthesis and frozen in liquid nitrogen. A total of 12 leaf samples were prepared, and they included 2 time points (10:00 and 12:00) of the two mulberry varieties; each time point has three biological replicates. The RNA of leaf samples was extracted using TRIzol reagent (Invitrogen, Thermo Fisher Scientific, USA) following the instruction. The quality of RNA sample was accessed with NanoDrop 2000 and Agilent 2100. After quality control, the qualified RNA was used for construction of Illumina RNA-seq libraries with Illunima TruSeq sample Prep Kit. The sequencing was performed with Illumina Hiseq Xten (San Diego, CA, USA) with 150 paired ends.

\section{RNA-seq data mining}

The raw reads generated from Illumina system were filtered and trimmed with fast QC and fastp. Subsequently, the obtained clean reads were mapped to the mulberry genome (NCBI genome accession number: 17692) with TopHat2. The FPKM values of gene were calculated using Cufflinks. The novel genes identified in this study were annotated with the public databases, including NCBI NR database, SwissProt, GO, KOG, Pfam, and KEGG.

The samples collected at two time points for E1 and H32 varieties were named as E1-10, E1-12, H32-10 and H32-12, respectively. A criterion of fold-change $\geq 2$ and false discovery rate $($ FDR $)<0.01$ was used to detect the differentially expressed genes (DEGs). The photosynthesisrelated DEGs were screened out from the two mulberry varieties with different yields. GO and KEGG pathway enrichment analyses of DEGs were performed with DAVID 6.7 tools.

\section{Validation of expression levels of DEGS}

The first-strand cDNA was synthetized using oligo-dT
(TaKaRa, Shiga, Japan). RT-qPCR was performed using Light Cycler 480 (Roche, Basel, Switzerland) in $20 \mu \mathrm{L}$ based on iTaq SYBR Green Mix (TakaRa, Shiga, Japan). The reaction conditions were $95^{\circ} \mathrm{C}$ for $3 \mathrm{~min}$, followed by 40 cycles of $94^{\circ} \mathrm{C}$ for $10 \mathrm{~s}, 55^{\circ} \mathrm{C}$ for $10 \mathrm{~s}$, and $72^{\circ} \mathrm{C}$ for $30 \mathrm{~s}$. The expression levels were calculated relative to the expression levels of $\beta$-actin and $G A D P H$ by using the $2^{-\Delta \Delta C t}$ method (Livak and Schmittgen 2001). Primers were designed using NCBI primer design program. The sequences of primers are shown in Table S1.

\section{Results}

Daily variation of photosynthesis of two mulberry varieties

The photosynthetic rate $(P \mathrm{n})$ of the two mulberry varieties from 6:00 to 18:00 showed a typical bimodal curve (Fig. 1A). The first peak appeared at 10:00, while the second peak appeared at 14:00. The $P$ n peaks of the leaves from E1 and $\mathrm{H} 32$ were 34.56 and $32.65 \mu \mathrm{mol} /\left(\mathrm{m}^{2} \cdot \mathrm{s}\right)$, respectively. Notably, the peak $P$ n of E1 was significantly higher than that of $\mathrm{H} 32(P<0.05)$. The $G$ s and $C$ i were gradually decreasing from 6:00 to 18:00 (Fig. 1B-C). The curve of transpiration rate ( $\mathrm{Tr}$ ) (transport resistance of $\mathrm{CO}_{2}$ and water) values was unimodal (Fig. 1D), which was increasing at 6:00 and reached a peak at 12:00 then gradually decreased. The Tr peaks of E1 and H32 leaves were 6.30 and 10.56 $\mathrm{mmol} /\left(\mathrm{m}^{2} \cdot \mathrm{s}\right)$, respectively. The peak of $\mathrm{Tr}$ in $\mathrm{H} 32$ was significantly higher than that in $\mathrm{E} 1(P<0.05)$.

\section{$\boldsymbol{P n}-\mathrm{PAR}$ and $\boldsymbol{P n}-\mathrm{Ci}$ response of the $\mathrm{E} 1$ and $\mathrm{H} 32$ varieties}

We found that carboxylation efficiency (CE) and $\mathrm{CO}_{2}$ saturation point (CSP) were higher in E1 rather than $\mathrm{H} 32$, while AQY, LCP, LSP, and CCP were higher in H32. The detailed summary is shown in Table 1. The LSP values of leaves in $\mathrm{H} 32$ and $\mathrm{E} 1$ were 1500 and $1400 \mu \mathrm{mol} /\left(\mathrm{m}^{2} \cdot \mathrm{s}\right)$, respectively, and the LCP values were 47.930 and 31.182 $\mu \mathrm{mol} /\left(\mathrm{m}^{2} \cdot \mathrm{s}\right)$, respectively, which indicated that the ability of accumulating photosynthate under weak light in E1 was stronger than that in H32. The CCP values of H32 and E1 were 74.618 and $68.724 \mu \mathrm{mol} / \mathrm{mol}$, respectively, indicating that E1 has higher utilization efficiency of low $\mathrm{CO}_{2}$ concentration. The CSP values in E1 and H32 were 1212.5 and $1162.5 \mu \mathrm{mol} / \mathrm{mol}$, respectively, implying that the $\mathrm{CO}_{2}$ concentration range utilized by E1 was greater than that of H32 (Cannell and Thornley 1998).

\section{Chlorophyll fluorescence parameters in E1 and H32 varieties}

As shown in Fig. 2A and Fig. 2B, no significant difference was observed in the Fo and Fm between the two mulberry varieties $(P>0.05)$ as well as the initial fluorescence between E1 and H32 (Fig. 2C). The ФPSII and $\mathrm{qP}$ were 
Li et al. / Intl J Agric Biol, Vol 25, No 4, 2021

Table 1: Comparison of major photosynthetic physiological parameters of the two mulberry varieties

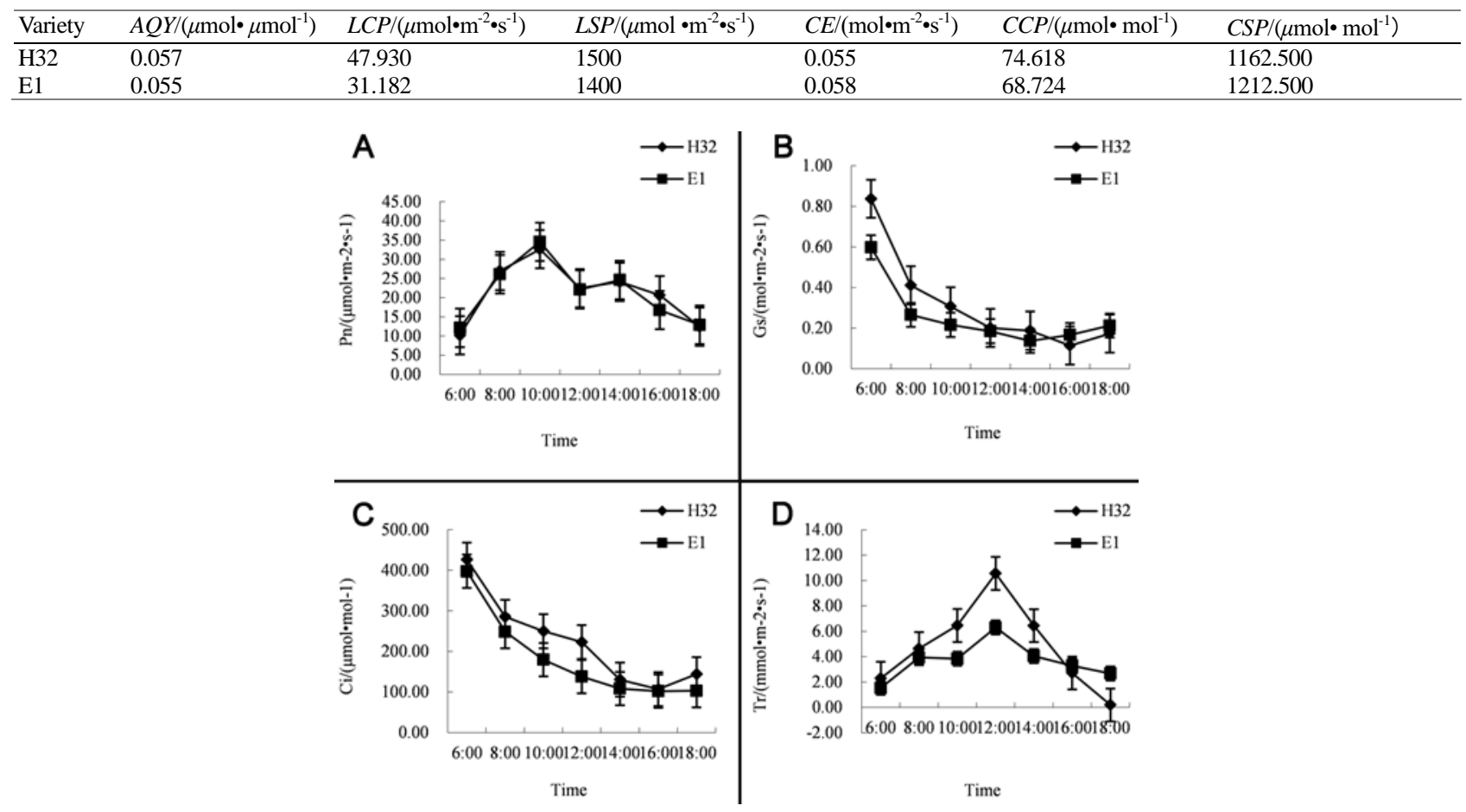

Fig. 1: Daily variation in gas exchange parameters of mulberry leaves. (A) Pn represents photosynthetic rate; (B) Gs represents stomatal conductance; $(\mathbf{C}) \mathrm{Ci}$ represents intercellular $\mathrm{CO}_{2}$ concentration; $(\mathbf{D}) \mathrm{Tr}$ represents transpiration rate; Error bars represent standard error. Different letters on error bars indicate significant differences at $P<0.01$. Symbols are the same in the Figs

greater in the leaves of $\mathrm{E} 1$ than in $\mathrm{H} 32(P<0.05)$ (Fig. 2DE), and the NPQ and ETR differed between the two different mulberry varieties. ФPSII and qP in the leaves of H32 were greater than those in E1 $(P<0.05)$ (Fig. $2 \mathrm{~F}-\mathrm{G})$.

\section{Chlorophyll content and activity of RuBP carboxylase}

After determining the photosynthesis and chlorophyll fluorescence parameters, leaf samples were collected to determine chlorophyll content and RuBP activity. We found that the contents of chlorophyll a, chlorophyll $b$, and total chlorophyll in $\mathrm{H} 32$ were significantly lower than those in E1 $(P<0.05)$ (Fig. 3A-B), which showed that the level of photosynthesis in E1 was much higher than in H32. Meanwhile, the RuBP activities in E1 and H32 were similar $(P>0.05)$.

\section{Gene expression profiling of the two different mulberry varieties}

Identification of differentially expressed genes (DEGs): A total of 23,136,096 and 27,147,665 clean reads were obtained for the two libraries (H32-10 and H32-12), respectively, in $\mathrm{H} 32$ variety. The numbers of clean reads obtained for two E1 libraries (E1-10 and E1-12) were $27,641,855$ and $30,242,866$, respectively. The clean reads were mapped to the reference genome, and the distribution of read coverage on the genome is shown in Fig. S1. The numbers of DEGs identified from the pairwise comparisons are shown in Fig. 4 and the 3,359 DEGs is listed in Table S1. A total of 507 DEGs between H32-10 and H32-12, and 297 DEGs were significantly up-regulated. Likewise, 585 DEGs were detected between E1-10 and E1-12, and 339 potential key genes were up-regulated. To investigate the difference of gene expression between two varieties, the comparison was also performed with H32-10 vs. E1-10. A total of 1,179 DEGs were detected between H32-10 and E1-10, including 781 up-regulated genes and 398 down-regulated genes. Furthermore, 1,085 DEGs (612 up-regulated and 473 downregulated genes) were detected in the comparison of $\mathrm{H} 32-12$ vs. E1-12.

\section{Functional analysis of DEGs}

The GO annotation of DEGs is shown in Fig. S2. The results of GO enrichment analyses showed that the DEGs were significantly enriched in some important GO terms, including branched-chain amino acid biosynthetic process, chalcone biosynthetic process, regulation of anthocyanin biosynthetic process, pectin catabolic process, organelle assembly, drug transmembrane transport, cellular biogenic amine biosynthetic process, defense response signaling pathway, resistance gene-dependent, and glycine catabolic process. 
Table 2: Photosynthesis-related metabolic pathways with significantly enriched DEGs

\begin{tabular}{llll}
\hline Comparison & KO ID & Pathway & Number of DEGs \\
\hline H32-10 vs. H32-12 & ko00710 & Carbon fixation in photosynthetic organisms & 5 \\
& ko01200 & Carbon metabolism & 7 \\
E1-10 vs. E1-12 & ko00860 & Porphyrin and chlorophyll metabolism & 4 \\
& ko00710 & Carbon fixation in photosynthetic organisms & 6 \\
& ko01200 & Carbon metabolism & 2 \\
H32-10 vs. E1-10 & ko00860 & Porphyrin and chlorophyll metabolism & 4 \\
& ko00710 & Carbon fixation in photosynthetic organisms & 9 \\
H32-12 vs. E1-12 & ko01200 & Carbon metabolism & 0 \\
& ko00710 & Porphyrin and chlorophyll metabolism & 2 \\
& ko01200 & Carbon fixation in photosynthetic organisms & 4 \\
\end{tabular}

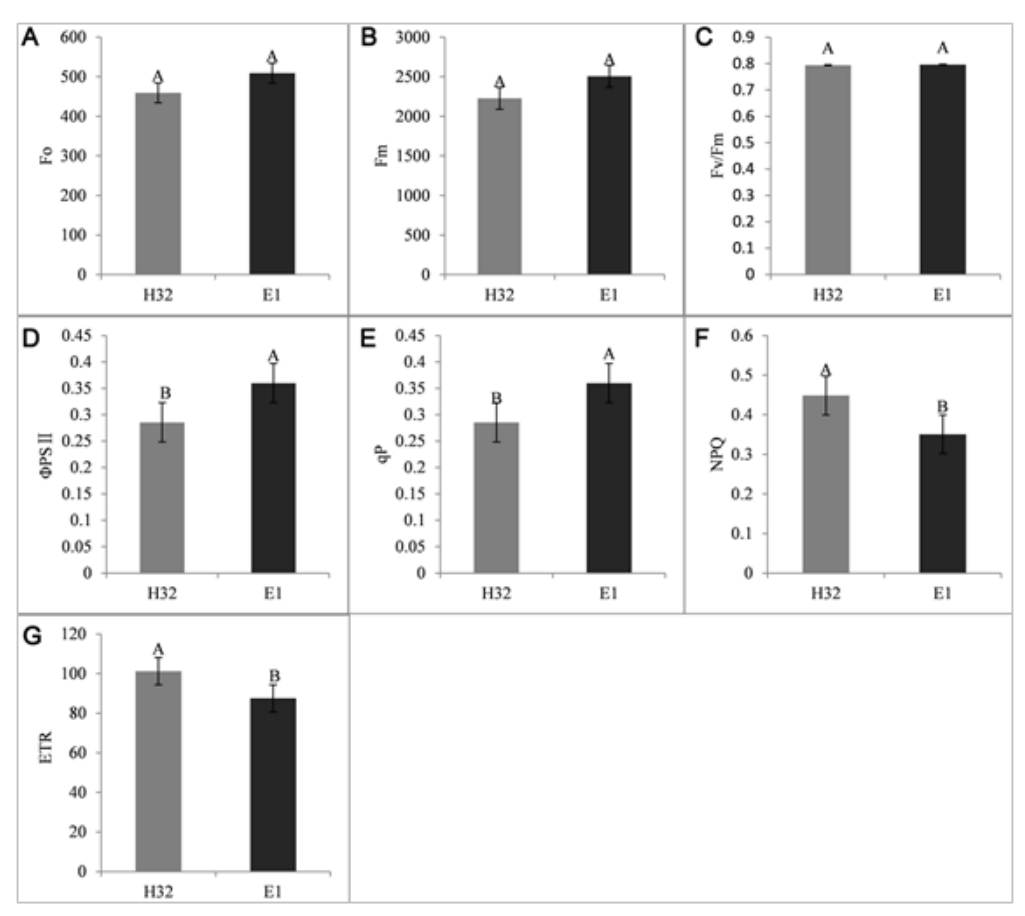

Fig. 2: Chlorophyll fluorescence parameters of mulberry leaves. (A) Fo represents minimum fluorescence. (B) Fm represents maximum fluorescence. (C) Fv/Fm represents the ratio of variable to maximal chlorophyll fluorescence. (D) $\Phi$ PSII represents actual photochemical quantum yield. (E) qP represents photochemical quenching. (F) NPQ represents amount of light energy. (G) ETR represents electron transport rate. Error bars represent standard error. Different letters on error bars indicate significant differences at $P<0.01$

To deeply explore the key genes and potential pathway involved in photosynthesis, the DEGs were classified into KEGG pathway, and the metabolic pathway was selected to focus on. The summary of DEGs related to metabolic pathway is shown in Fig. S3. In detail, a total of 232, 190, 380 and 334 DEGs were involved in metabolic pathways in $\mathrm{H} 32-10$ vs. H32-12, E1-10 vs. E1-12, H32-10 vs. E1-10, and H32-12 vs. E1-12, respectively. More importantly, the DEGs related to three photosynthesis-related metabolic pathways, i.e., carbon fixation in photosynthetic organisms, carbon metabolism, porphyrin and chlorophyll metabolism, were summarized (Table 2). The expression levels of photosynthesis- related genes in metabolic pathways are shown in Table 3. The LOC4331897 (1,4-dihydroxy-2naphthoate polyprenyltransferase), which is involved in carbon fixation in photosynthetic organisms, was significantly up-regulated in E1-12 compared with E1-10, but interestingly, it was not up-regulated in H32-10 vs. H3212. As a key factor in porphyrin and chlorophyll metabolism, the transcripts of chlorophyllase were down-regulated in E1$10 \mathrm{vs}$. E1-12, but they were not differentially expressed in H32-10 vs. H32-12. Meanwhile, some transcripts of genes participating in carbon fixation were differentially expressed between $\mathrm{H} 32$ and $\mathrm{E} 1$ varieties. For instance, the transcripts of pyruvate phosphate dikinase were down-regulated and NADP-dependent malic enzyme was up-regulated in H32$12 v s$. E1-12. Moreover, the similar dynamics of these genes were observed in the H32-10 vs. E1-10. As an important gene in the porphyrin and chlorophyll metabolism, the transcripts of chlorophyllase-1 were up-regulated in H32-12 
Li et al. / Intl J Agric Biol, Vol 25, No 4, 2021

Table 3: Summary of photosynthesis-related genes in the metabolic pathways

\begin{tabular}{|c|c|c|c|c|c|c|}
\hline Comparison & Biological process & KO ID & Gene & Up/Down & FDR & Log2FC \\
\hline \multirow[t]{16}{*}{ E1-10 vs. E1-12 } & $\begin{array}{l}\text { carbon fixation in photosynthetic } \\
\text { organisms }\end{array}$ & ko00710 & Phosphoenol pyruvate carboxylase 2 & Down & $1.01 \mathrm{E}-21$ & -1.07455 \\
\hline & & K02548 & 1,4-dihydroxy-2-naphthoate polyprenyltransferase & Up & $2.58 \mathrm{E}-13$ & 1.154945 \\
\hline & & ko00710 & Fructose-bisphosphate aldolase 1 & Down & 4.83E-12 & -1.18374 \\
\hline & & ko00710 & Fructose-bisphosphate aldolase 2 & Down & $3.19 \mathrm{E}-27$ & -1.5095 \\
\hline & & ko00710 & Fructose-1,6-bisphosphatase & Down & $5.89 \mathrm{E}-20$ & -1.00595 \\
\hline & carbon metabolism & ko01200 & Phosphoenolpyruvate carboxylase 2 & Down & $1.01 \mathrm{E}-21$ & -1.07455 \\
\hline & & ko01200 & Fructose-bisphosphate aldolase 1 & Down & 4.83E-12 & -1.18374 \\
\hline & & ko01200 & Fructose-bisphosphate aldolase 2 & Down & $3.19 \mathrm{E}-27$ & -1.5095 \\
\hline & & ko01200 & Fructose-1,6-bisphosphatase & Down & $5.89 \mathrm{E}-20$ & -1.00595 \\
\hline & & ko01200 & D-3-phosphoglycerate dehydrogenase & Down & $3.47 \mathrm{E}-23$ & -1.09837 \\
\hline & & ko01200 & glucose-6-phosphate 1-dehydrogenase & Up & $3.76 \mathrm{E}-30$ & 1.452002 \\
\hline & & K02548 & 1,4-dihydroxy-2-naphthoate polyprenyltransferase & Up & $2.58 \mathrm{E}-13$ & 1.154945 \\
\hline & porphyrin and chlorophyl metabolism & ko00860 & Chlorophyllase & Down & 0.000149 & -1.96728 \\
\hline & & ko00860 & Chlorophyllase-1 & Up & $1.51 \mathrm{E}-18$ & 2.689146 \\
\hline & & ko00860 & Chlorophyllase-2 & Up & $4.38 \mathrm{E}-11$ & 1.741928 \\
\hline & & ko00860 & Protochlorophyllide reductase & Up & $2.42 \mathrm{E}-25$ & 1.722631 \\
\hline \multirow[t]{12}{*}{$\mathrm{H} 32-10$ vs. H32-12 } & carbon fixation in photosynthetic organisms & ko00710 & Fructose-bisphosphate aldolase 1 & Down & $1.60 \mathrm{E}-06$ & -1.18952 \\
\hline & & ko00710 & Fructose-bisphosphate aldolase 2 & Down & 3.19E-27 & -1.5095 \\
\hline & & ko00710 & Transketolase & Down & 0.00013 & -1.00502 \\
\hline & & ko00710 & Fructose-1,6-bisphosphatase & Down & 0.000597 & -1.15076 \\
\hline & carbon metabolism & ko01200 & Fructose-bisphosphate aldolase, cytoplasmic isozyme 1 & Down & $1.60 \mathrm{E}-12$ & -1.18952 \\
\hline & & ko01200 & putative fructose-bisphosphate aldolase 2 & Down & $9.29 \mathrm{E}-10$ & -1.27176 \\
\hline & & ko01200 & Fructose-1,6-bisphosphatase & Down & 0.000597 & -1.15076 \\
\hline & & ko01200 & D-3-phosphoglycerate dehydrogenase & Down & 7.94E-09 & -1.11896 \\
\hline & & ko01200 & glucose-6-phosphate 1-dehydrogenase, chloroplastic-like & Up & $1.47 \mathrm{E}-23$ & 1.790828 \\
\hline & & ko01200 & Transketolase & Down & 0.00013 & -1.00502 \\
\hline & porphyrin and chlorophyl metabolism & ko00860 & Chlorophyllase-1 & Up & $7.25 \mathrm{E}-05$ & 1.28331 \\
\hline & & ko00860 & Protochlorophyllide reductase & Up & $6.95 \mathrm{E}-24$ & 1.837399 \\
\hline \multirow[t]{7}{*}{ H32-12 vs. E1-12 } & carbon fixation in photosynthetic organisms & ko00710 & Pyruvate phosphate dikinase & Down & $1.31 \mathrm{E}-29$ & -1.37241 \\
\hline & & ko00710 & NADP-dependent malic enzyme & Up & $5.70 \mathrm{E}-11$ & 1.123941 \\
\hline & carbon metabolism & ko01200 & D-3-phosphoglycerate dehydrogenase & Down & 6.67E-06 & -1.775 \\
\hline & & ko01200 & NADP-dependent malic enzyme & Up & $5.70 \mathrm{E}-11$ & 1.123941 \\
\hline & & ko01200 & Pyruvate phosphate dikinase & Down & $1.31 \mathrm{E}-29$ & -1.37241 \\
\hline & & ko01200 & hypothetical protein L484_016723 & Up & $1.04 \mathrm{E}-10$ & \\
\hline & porphyrin and chlorophyl metabolism & ko00860 & Chlorophyllase-1 & Up & $2.28 \mathrm{E}-06$ & -2.37285 \\
\hline \multirow[t]{12}{*}{$\mathrm{H} 32-10$ vs. E1-10 } & carbon fixation in photosynthetic organisms & ko00710 & Pyruvate phosphate dikinase & Down & 0.000126 & -1.06521 \\
\hline & & ko00710 & Photosystem Q(B) protein & Down & 0.004188 & -1.04555 \\
\hline & & ko00710 & NADP-dependent malic enzyme & Up & $1.16 \mathrm{E}-07$ & 1.072298 \\
\hline & & ko00710 & Phosphoenolpyruvate carboxylase, housekeeping isozyme & Up & $1.23 \mathrm{E}-09$ & 1.119376 \\
\hline & carbon metabolism & ko01200 & hypothetical protein L484_016723 & Up & 7.31E-08 & 6.315284 \\
\hline & & ko01200 & GRAS domain family, Scarecrow-like protein & Up & 0.003807 & 1.715487 \\
\hline & & ko01200 & Pyruvate, phosphate dikinase & Down & 0.000126 & -1.06521 \\
\hline & & ko01200 & Alcohol dehydrogenase-like 2 & Up & 0.007645 & 1.411398 \\
\hline & & ko01200 & 2-oxoglutarate dehydrogenase & Up & $3.84 \mathrm{E}-10$ & 1.150373 \\
\hline & & ko01200 & NADP-dependent malic enzyme & Up & $1.16 \mathrm{E}-07$ & 1.072298 \\
\hline & & ko01200 & Phosphoenolpyruvate carboxylase, housekeeping isozyme & Up & $1.23 \mathrm{E}-09$ & 1.119376 \\
\hline & & ko01200 & Pyruvate kinase isozyme $\mathrm{G}$ & Up & $2.30 \mathrm{E}-07$ & 1.330851 \\
\hline
\end{tabular}

porphyrin and chlorophyl metabolism

vs. E1-12, whereas they were not detected in H32-10 vs. E1-10.

\section{Screening of novel photosynthesis-related genes}

We compared the transcripts to the reference genome identified the genes which had not been annotated in the genome, the genes with length $>150 \mathrm{bp}$ and more than one exon were remained. According to the functional annotation of 3,359 DEGs, 10 novel DEGs related to photosynthesis were screened out. Of these, 3 genes were down-regulated in H32-10 vs. H32-12 and E1-10 vs. E1-12. Also, 2 upregulated genes were found in $\mathrm{H} 32-10$ vs. E1-10 and H32-12 vs. E1-12. The details of 10 novel genes are shown in Table 4. Five novel genes were differentially expressed in H32-10 vs. H32-12 and E1-10 vs. E1-12, and 3 of them, annotated into carbohydrate transport and metabolism, defense mechanisms, metabolites biosynthesis, and transport and catabolism, were down-regulated in $\mathrm{H} 32-10$ and E1-10, while genes (i.e., glucose-6-phosphate 1-dehydrogenase, CHUP1, and chloroplastic were up-regulated. In the comparisons of E1 and H32 varieties, the novel genes annotated into GTP diphosphokinase RSH3, chloroplastic, translocase of chloroplast 34, chloroplastic, and cytochrome P450 71A2 were down-regulated in H32. These novel genes may play important roles in the net photosynthetic rates between E1 
Table 4: Dynamics of candidate genes related to photosynthesis at H32-10 vs. H32-12 and E1-10 vs. E1-12

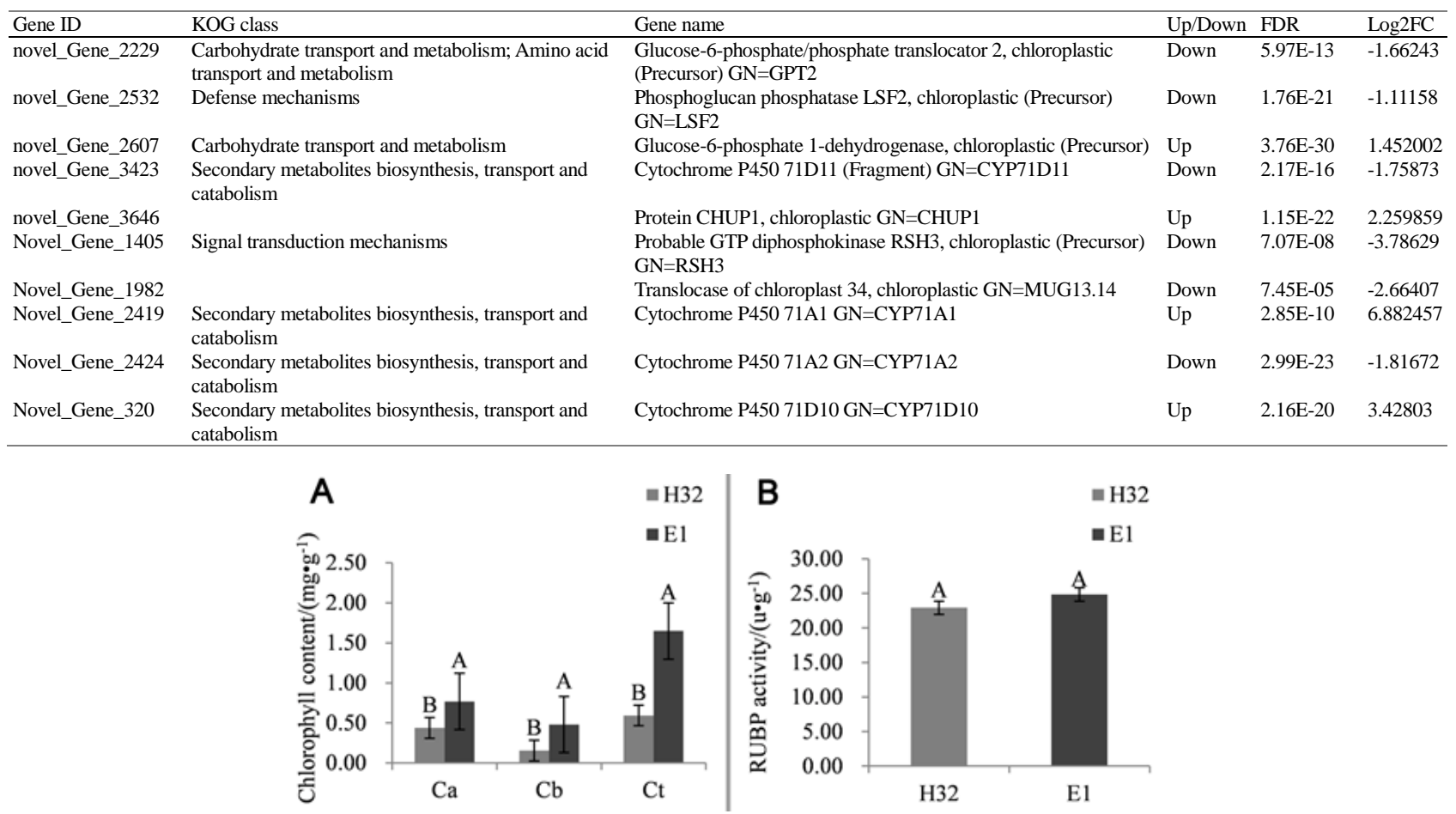

Fig. 3: Comparison of chlorophyll content and RUBP activity in mulberry leaves. (A) Ca represents leaf chlorophyll a content, Cb represents lesfchlouophyll b content, Ct represents leaf total chlorophyll content. (B) RUBP activity represents Ribulose 1,5-bisphosphate carboxylase activity. Error bars represent standard error. Different letters on error bars indicate significant differences at $P<0.01$

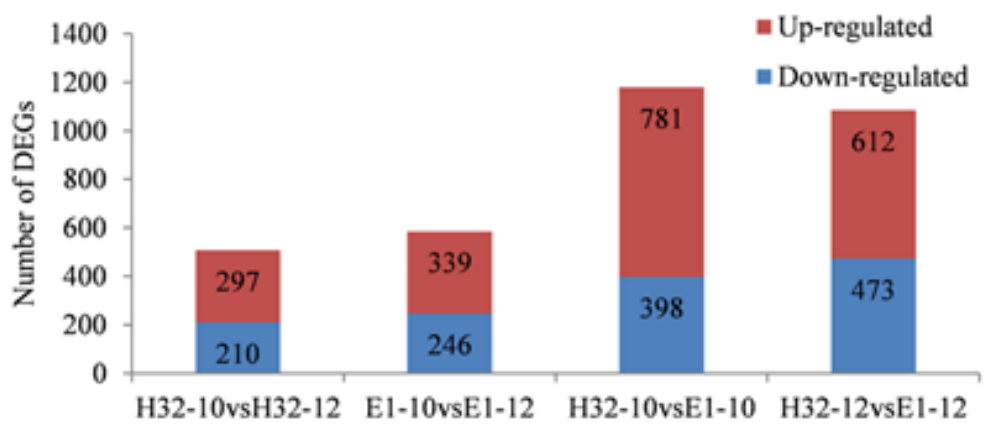

Fig. 4: Statistics of differentially expressed genes in the pairwise comparisons

and $\mathrm{H} 32$ at different and the same time intervals.

\section{Validation of RNA-seq data}

The expression levels of the 10 novel genes were investigated using RT-qPCR method (Table S2). Five of them (Fig. 5A-E) except Novel_gene_3423 showed significant difference between the time points $(P<0.05)$, and the other five novel genes (Fig. 5F-J) were differentially expressed between the varieties $(P<0.05)$, which coincides with the RNA-seq data. It indicated that the gene expression levels determined by RNA-seq data were reliable in this study.

\section{Discussion}

Mulberry is an economic food crop for the domesticated silkworm more than 5000 years (Rudramuni et al. 2019). The mulberry varieties (E1 and H32) are two representative varieties which have been widely planted in China, especially in Yangtze River basin. In this study, we comprehensively investigated the daily variations of photosynthetic rate from 6:00 to 18:00 in the E1 and H32. The gas exchange parameters showed obvious difference between the two mulberry varieties, and an increased rate of photosynthesis occurred in E1 variety. According to the results of $P$ n-PAR and $P$ n- $C$ i response, it indicated that E1 


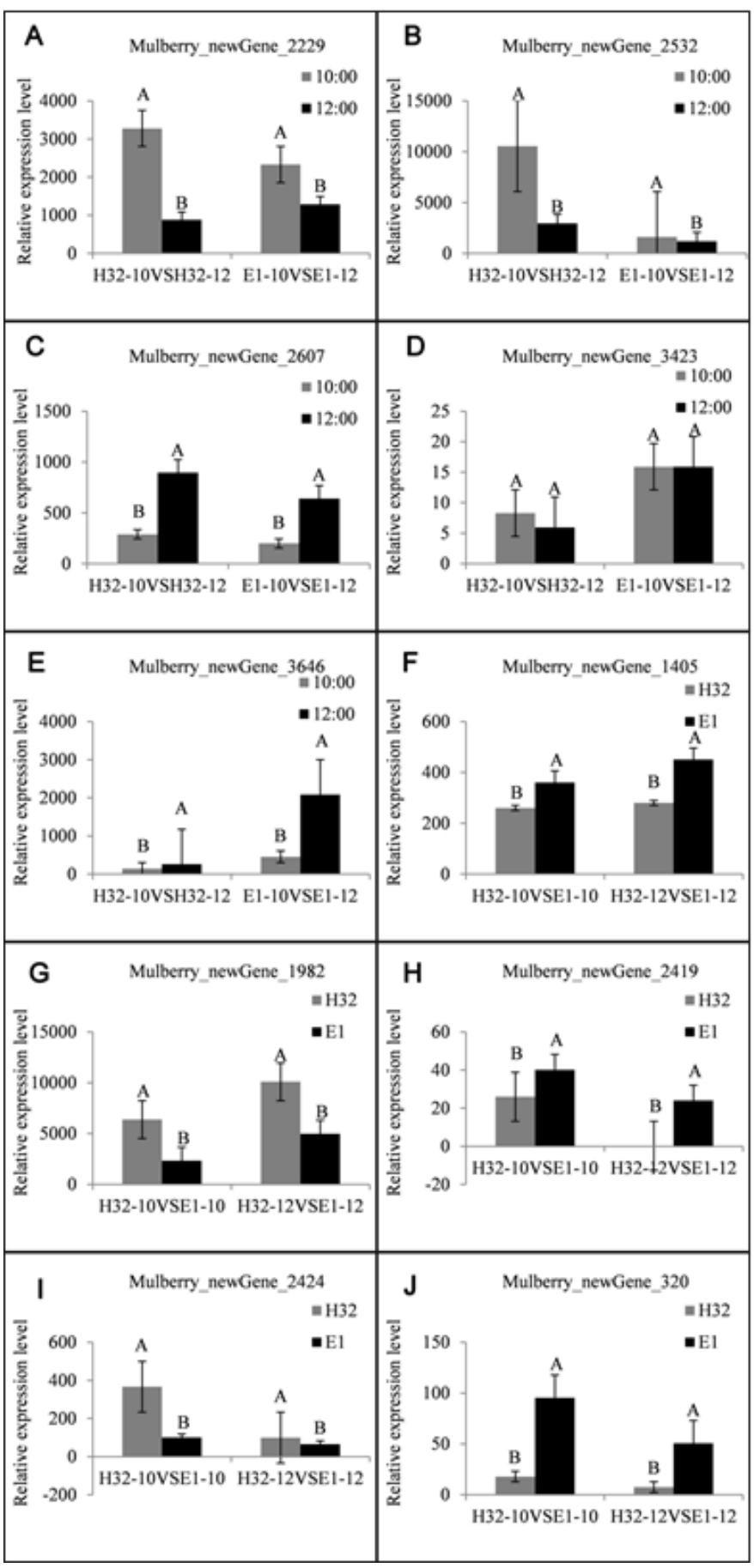

Fig. 5: Quantitative RT-PCR of ten candidate genes related to photosynthesis

had greater photosynthetic capacity than H32 and showed higher potential in accumulating photosynthetic products under weak light intensity. Based on the theory of Farquhar and Sharkey (1982), a higher $G$ s is a prerequisite for the higher $\mathrm{Pn}$. The enhanced $\mathrm{G}$ s can promote $\mathrm{CO}_{2}$ movement in the stomatal cavity in response to $\mathrm{CO}_{2}$ concentration. It has been demonstrated that the primary determinant of crop yield is the cumulative rate of photosynthesis (Lawson et al.
2012; Simkin et al. 2019), which could account for the difference of yield between two varieties.

ФPSII is an important indicator of plant photosynthetic capacity and can reflects the proportion of the excitation energy used for the photochemical pathways in the total excitation energy of PSII (Liu et al. 2018). qP, a photochemical quenching coefficient, reflects the amount of light energy absorbed by the PSII antenna pigment and used 
for the photochemical reaction. In this study, both of ФPSII and $\mathrm{qP}$ in $\mathrm{E} 1$ were higher than those in $\mathrm{H} 32$, indicating that E1 reflects the increased demand in the Calvin cycle for ATP and NADPH, and an increase in leaves qP indicated an up-regulation of the rate of consumption of reductants and ATP (Lim et al. 2020). Meanwhile, the contents of chlorophyll a, chlorophyll b, and chlorophyll in H32 were lower than E1. Overall, most of the estimated values of photosynthetic characters were significantly larger in E1 than in H32, which coincided with the previous study (Deng et al. 2012).

Gene regulation plays important roles on photosynthesis in plants, particularly the genes in metabolic pathway mediating the efficiency of photosynthesis. In the previous studies (Ding et al. 2013; Ashraf and Harris 2013), some important genes involved in photosynthesis have been identified, such as $S p 1, P e p c, R b c L$ and $P p d k$. In this study, a number of DEGs was detected between the two varieties. Of these, the DEGs in metabolic pathway were screened out, and three photosynthesis-related metabolic pathways, including carbon fixation, carbon metabolism, and porphyrin and chlorophyll metabolism were detected. Most importantly, the DEGs related to carbon fixation and metabolism was significantly up-regulated in E1 compared with H32. For instance, the gene Pepc (Phosphoenolpyruvate carboxylase), which is essential to the production of carbon skeletons for amino acid biosynthesis in plants (Heyduk et al. 2019). PEPC is one of the key proteins of photosynthetic pathway which catalyses the initial fixation of atmospheric $\mathrm{CO}_{2}$. Bandyopadhyay et al. (2007) introduced intact maize pepc gene in indica rice by biolistic transformation and found that the photosynthesis rate of rice was enhanced in high temperature conditions. Likewise, the up-regulation of NADP-dependent malic enzyme (NADP-ME) in E1 was detected, which catalyzes the oxidative decarboxylation of malate to generate pyruvate, $\mathrm{CO}_{2}$ and NADPH. In plants, the photosynthetic NADP-MEs supply $\mathrm{CO}_{2}$ for carbon fixation in the bundle sheath chloroplasts of $\mathrm{C} 4$ plants and the cytosol of crassulacean acid metabolism (CAM) plants (Alvarez et al. 2019; Chen et al. 2019).

\section{Conclusion}

In the comparison of E1 and H32, many DEGs related to the porphyrin and chlorophyll metabolism were identified, which is related to the synthesis, utilization and degradation of porphyrin and chlorophyll, a group of green magnesiumcontaining porphyrin derivatives occurring in photosynthetic plants. Besides, some novel DEGs were identified in this study, which were not annotated in the reference genome. These novel genes were annotated against the public databases, and we found most of them could match the homologue genes in the databases and a few novel genes were involved in photosynthesis of mulberry. These novel genes should be deeply explored in our further study.

\section{Acknowledgement}

The study was funded by National Fund for Modern Agricultural Industry Technology System Construction (No: CARS-18-ZJ0208).

\section{Author Contributions}

Conceived and designed the experiments: Yong $\mathrm{Li}$, Chuxiong Zhuang and Wen Deng; Performed the experiments: Yong Li,Cui Yu and Rongli Mo; Analysed the data: Yong Li,Chao Xiong and Zhixian Zhu; Contributed reagents/materials/analysis tools: Yong Li and Xingming $\mathrm{Hu}$; Contributed to the writing of the manuscript: Yong Li All of the authors reviewed the manuscript

\section{Conflict of Interest}

The authors of this article have no conflict of interest

\section{Data Availability Declaration}

The authors declare that data reported in this article are available with the corresponding author and will be produced on demand

\section{References}

Agastian P, S Kingsley, M Vivekanandan (2000). Effect of salinity on photosynthesis and biochemical characteristics in mulberry genotypes. Photosynthetica 38:287-290

Alvarez CE, A Bovdilova, A Höppner, CC Wolff, M Saigo, F Trajtenberg, MJ Lercher (2019). Molecular adaptations of NADP-malic enzyme for its function in C4 photosynthesis in grasses. Nat Plants 5:755-765

Ashraf M, PJ Harris (2013). Photosynthesis under stressful environments: An overview. Photosynthetica 51:163-190

Bandyopadhyay A, K Datta, J Zhang, W Yang, S Raychaudhuri, M Miyao, SK Datta (2007). Enhanced photosynthesis rate in genetically engineered indica rice expressing pepc gene cloned from maize. Plant Sci 172:1204-1209

Brouwer B, A Ziolkowska, M Bagard, O Keech, P Gardestrom (2012). The impact of light intensity on shade-induced leaf senescence. Plant Cell Environ 35:1084-1098

Cannell MGR, JHM Thornley (1998). Temperature and $\mathrm{CO}_{2}$ Responses of Leaf and Canopy Photosynthesis: A clarification using the nonrectangular hyperbola model of photosynthesis. Ann Bot 82:883-892

Chaitanya KV, PP Jutur, D Sundar, AR Reddy (2003). Water stress effects on photosynthesis in different mulberry cultivars. Plant Growth Regul 40:75-80

Chen F, L Chen, H Zhao, H Korpelainen, C Li (2010). Sex-specific responses and tolerances of Populus cathayana to salinity. Physiol Plantarum 140:163-173

Chen Q, B Wang, H Ding, J Zhang, S Li (2019). The role of NADP-malic enzyme in plants under stress. Plant Sci 281:206-212

Dai F, G Luo, Z Li, X Wei, Z Wang, S Lin, C Tang (2020). Physiological and transcriptomic analyses of mulberry (Morus atropurpurea) response to cadmium stress. Ecotoxicol Environ Saf 205:111298

Dai F, Z Wang, G Luo, C Tang (2015). Phenotypic and transcriptomic analyses of autotetraploid and diploid mulberry (Morus alba L.). Intl J Mol Sci 16:22938-22956

Demmig B, K Winter, A Kruger, FC Czygan (1987). Photoinhibition and zeaxanthinformationinintactleaves: A possible role of the xanthophyll cyclein the dissipation of excess light energy. Plant Physiol 84:218-224 
Deng Y, J Li, S Wu, Y Zhu, Y Chen, F He (2006). Integrated nr database in protein annotation system and its localization. Comput Eng 32:71-72

Ding ZS, SH Huang, BY Zhou, XF Sun, M Zhao (2013). Over-expression of phosphoenolpyruvate carboxylase cDNA from C4 millet (Seteria italica) increase rice photosynthesis and yield under upland condition but not in wetland fields. Plant Biotechnol Rep 7:155-163

Farquhar GD, TD Sharkey (1982). Stomatal Conductance and Photosynthesis. Annu Rev Plant Physiol 33:317-345

Feng L, MA Raza, Z Li, Y Chen, MHB Khalid, J Du, Z Zhang (2019). The influence of light intensity and leaf movement on photosynthesis characteristics and carbon balance of soybean. Front Plant Sci 9; Article 1952

Genty B, JM Briantais, NR Baker (1989). The relationship between the quantum yield of photosynthetic electron transport and quenching of chlorophyll fluorescence. Biochim Biophys Acta 990:87-92

Heyduk K, JJ Moreno-Villena, IS Gilman, PA Christin, EJ Edwards (2019). The genetics of convergent evolution: Insights from plant photosynthesis. Nat Rev Genet 20:485-493

Kubien DS, CM Brown, HJ Kane (2011). Quantifying the amount and activity of Rubisco in leaves. Meth Mol Biol 684:349-362

Lawson T, DM Kramer, CA Raines (2012). Improving yield by exploiting mechanisms underlying natural variation of photosynthesis. Curr Opin Biotechnol 23:215-220

Li Y, C Ye, W Deng, YU Cui, X Hu, C Xiong, B Peng, H Shi (2014). Effects of spring-cutting on photosynthetic physiological parameters of Morus alba L. cv. E-sang No. 1. Hubei Agric Sci 53:4885-4891

Lim SL, CP Voon, X Guan, Y Yang, P Gardeström, BL Lim (2020). In planta study of photosynthesis and photorespiration using NADPH and $\mathrm{NADH} / \mathrm{NAD}^{+}$fluorescent protein sensors. Nat Commun 11; Article 3238

Liu Y, T Wang, S Fang, M Zhou, J Qin (2018). Responses of morphology, gas exchange, photochemical activity of photosystem II, and antioxidant balance in Cyclocarya paliurus to light spectra. Front Plant Sci 9:1704-1718

Livak KJ, TD Schmittgen (2001). Analysis of relative gene expression data using real-time quantitative PCR and the 2(-Delta Delta $\mathrm{C}(\mathrm{T})$ ) Method. Methods 25:402-408

Nemali K, MWV Iersel (2019). Relating whole-plant photosynthesis to physiological acclimations at leaf and cellular scales under drought stress in bedding plants. J Amer Soc Hortic 144:201-208

Peng X, L Teng, X Yan, M Zhao, S Shen (2015). The cold responsive mechanism of the paper mulberry: Decreased photosynthesis capacity and increased starch accumulation. BMC Genomics 16; Article 898
Peñuelas J, I Filella, J Llusià, D Siscart, J Piñol (1998). Comparative field study of spring and summer leaf gas exchange and photobiology of the Mediterranean trees Quercus ilex and Phillyrea latifolia. J Exp Bot 49:229-238

Porra RJ, WA Thompson, PE Kriedemann (1989). Determination of accurate extinction coefficients and simultaneous equations for assaying chlorophylls a and $\mathrm{b}$ extracted with four different solvents: Verification of the concentration of chlorophyll standards by atomic absorption spectroscopy. Biochim Biophys Acta 975:384-394

Ramanjulu S, N Sreenivasulu, C Sudhakar (1998). Effect of Water Stress on Photosynthesis in Two Mulberry Genotypes with Different drought Tolerance. Photosynthetica 35:279-283

Rudramuni K, BK Neelaboina, MN Shivkumar, SR Chowdhury (2019). Scope for Region and Season Specific Mulberry Silkworm (Bombyx mori L.) in Temperate Regions of Jammu and Kashmir. Res J Agric Sci 10:809-814

Ryu Y, JA Berry, DD Baldocchi (2019). What is global photosynthesis? History, uncertainties and opportunities. Remot Sens Environ 223:95-114

Shen H, Y Tang, H Muraoka, I Washitani (2008). Characteristics of leaf photosynthesis and simulated individual carbon budget in Primula nutans under contrasting light and temperature conditions. J Plant Res 121:191-200

Simkin AJ, PE López-Calcagno, CA Raines (2019). Feeding the world: Improving photosynthetic efficiency for sustainable crop production. $J$ Exp Bot 70:1119-1140

Tezara W, VJ Mitchell, SD Driscoll, DW Lawlor (1999). Water stress inhibits plant photosynthesis by decreasing coupling factor and ATP. Nature 401:914-917

Wang D, L Zhao, D Wang, J Liu, X Yu, Y Wei, Z Ouyang (2018). Transcriptome analysis and identification of key genes involved in 1deoxynojirimycin biosynthesis of mulberry (Morus alba L.). Peer J 6; Article e5443

Wu Y, W Gong, W Yang (2017). Shade inhibits leaf size by controlling cell proliferation and enlargement in soybean. Sci Rep 7:9259-9268

Ye C, W Deng, W Ye (2010). Study on the cultivation of mulberry variety Ersang No. 1 and its application. Hubei Agric Sci 49:109-111

Yu C, S Huang, X Hu, W Deng, C Xiong, C Ye, B Peng (2013). Changes in photosynthesis, chlorophyll fluorescence, and antioxidant enzymes of mulberry (Morus sspp.) in response to salinity and hightemperature stress. Biologia 68:404-413

Zhou J, Y Li, C Yu, R Mo Z Zhu, Z Dong, X Hu, W Deng (2019). Effects of photosynthetic characteristics and sugar metabolism of fruit mulberry in different breeding methods. Hubei Agric Sci 58:89 